\title{
Adaptive Streaming Playback Statistics Dataset
}

\author{
Thiago Teixeira \\ Brightcove, Inc. \\ Boston, Massachusetts, USA \\ tteixeira@brightcove.com
}

\author{
Bo Zhang \\ Brightcove, Inc. \\ Boston, Massachusetts, USA \\ bzhang@brightcove.com
}

\author{
Yuriy Reznik \\ Brightcove, Inc. \\ Seattle, WA, USA \\ yreznik@brightcove.com
}

\begin{abstract}
We propose dataset capturing statistics of several large-scale realworld streaming events, delivering videos to different devices (TVs, desktops, mobiles, tablets, etc.), and over different networks (from 2.5G, 3G and other early generation mobile networks to 5G and broadband). The data we capture include network-related statistics, playback statistics (session- and player-event-level), and characteristics of the encoded streams. Such data should enable a broad level of possible applications and uses in the research community: from analysis of the effectiveness of algorithms in streaming players to studies of QoE metrics, and end-to-end system optimizations. Examples of such possible studies based on the proposed datasets are also provided.
\end{abstract}

\section{CCS CONCEPTS}

- Multimedia information systems $\rightarrow$ Multimedia streaming

\section{KEYWORDS}

Media streaming, HTTP Adaptive Streaming, DASH, HLS, ABR Algorithms, Playback Statistics, Datasets

\section{ACM Reference format:}

Thiago Teixeira, Bo Zhang, and Yuriy Reznik. 2021. Adaptive Streaming Playback Datasets. In Proceedings of ACM Multimedia Systems conference (MMSys'21). ACM, New York, NY, USA, 6 pages. https://doi.org/10.1145/1234567890

\section{INTRODUCTION}

During last two decades Internet streaming has evolved from a pioneering concept to mainstream technology used for delivery of media content. An important step in this evolution was the invention of the concept of adaptive bit-rate (ABR) streaming [1-3]. The key idea was to encode content at multiple bitrates, and then make the delivery system switch between such encoded streams adaptively, as needed to match network bandwidth,

Permission to make digital or hard copies of all or part of this work for personal or classroom use is granted without fee provided that copies are not made or distributed for profit or commercial advantage and that copies bear this notice and the full citation on the first page. Copyrights for components of this work owned by others than ACM must be honored. Abstracting with credit is permitted. To copy otherwise, or republish, to post on servers or to redistribute to lists, requires prior specific permission and/or a fee. Request permissions from permissions@acm.org.

MMSys 21, September 28-October 1, 2021, Istanbul, Turkey

(C) 2021 Association for Computing Machinery.

ACM ISBN 978-1-4503-8434-6/21/09.

https://doi.org/10.1145/3458305.3478444 available at each point in time. Likely first commercial system that implemented it was RealSystem G2, launched around 1998 [3]. The ABR mechanism has since become widely adopted and provides basis for today's HTTP-based adaptive streaming system, such as HLS and DASH [4,5].

In Figure 1, we show a conceptual diagram of a modern-era HTTP-based streaming system. The input video feed is typically provided over a contribution link, connecting it to the streaming transcoder. The transcoder then produces several streams with different bitrates and resolutions and places them on the origin server. The distribution is handled by a CDN. And the receiving devices use special software called streaming client that makes decisions about which streams to load at each point in time. The switching is enabled by coordinated insertion of IDR frames in all streams at certain periodicity, and then creating an index or manifest file enabling the client to pull streams at IDR boundaries. The streams may also be segmented and placed on origin servers as sequences of files representing each encoded segment

Importantly, Figure 1 also shows an analytics system collecting player logs and other statistics is the system. In practice, such statistics are used for a variety of business reasons. For example, they can be used to monitor system operation and rapidly respond to problems if they are detected. QoE measurement and monitoring may also be part of business operation. Analytics data may also be used to understand which videos have been delivered/watched most often, or the geographic distribution of viewers watching them. They may also be used for tracking and monetization of video impressions delivered by the inserted ad content.

But there are also some advanced uses of analytics data that we believe belong to the domain of research and progressive work on modeling and improving the performance of streaming systems. For example, by considering that modern-era streaming systems deliver streams to a variety of devices, with a variety of streaming client implementations, and with different constraints in terms of screen sizes, codecs, DRM, and file-format support, we are bound to discover that behavior of such system is quite complex, and cannot be easily predicted by assuming simple bitrate-based adaptation logic. Some clients may base their stream selection decisions on additional factors: the size of the player window, CPU capabilities of the device, user behavior, etc. By looking at playback statistics we may also discover some inefficiencies in the system. For example, some clients may be loading much data ahead and then discarding them when the user stops watching the content. As illustrated in Figure 2, some clients may also be loading and then discarding some segments when switching between streams. There could also be also many other suboptimalities in players' 


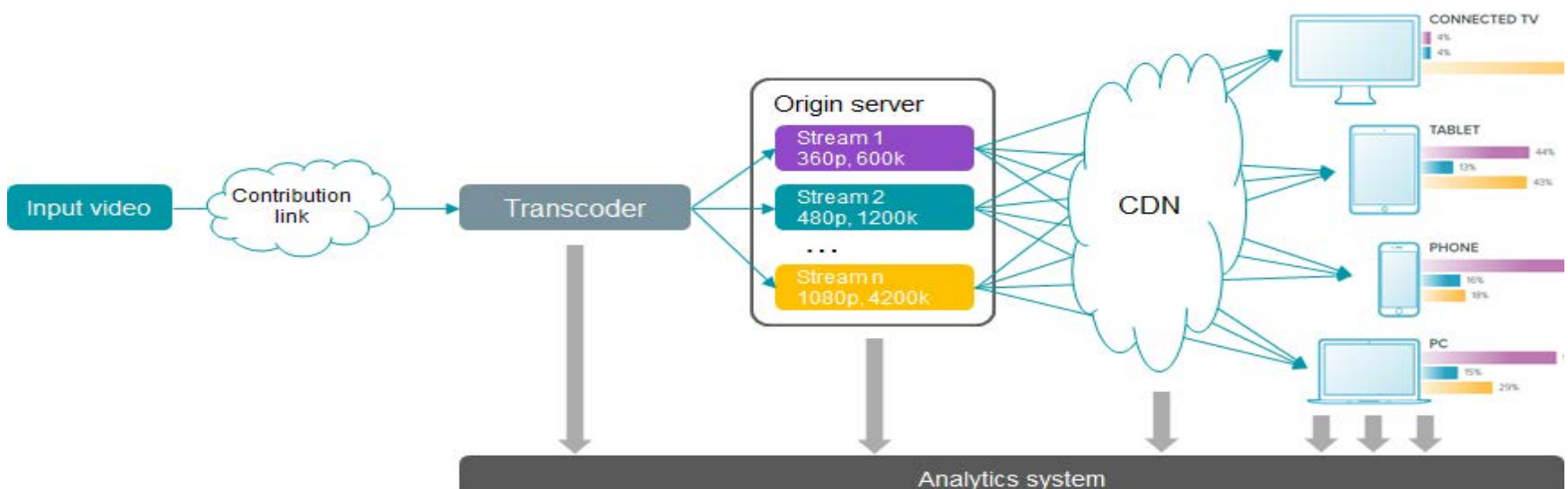

Figure 1: Conceptual diagram of an HTTP-based adaptive bitrate streaming system with analytics processing.

Bandwidth fluctuations, segment loading \& rendition switching events:

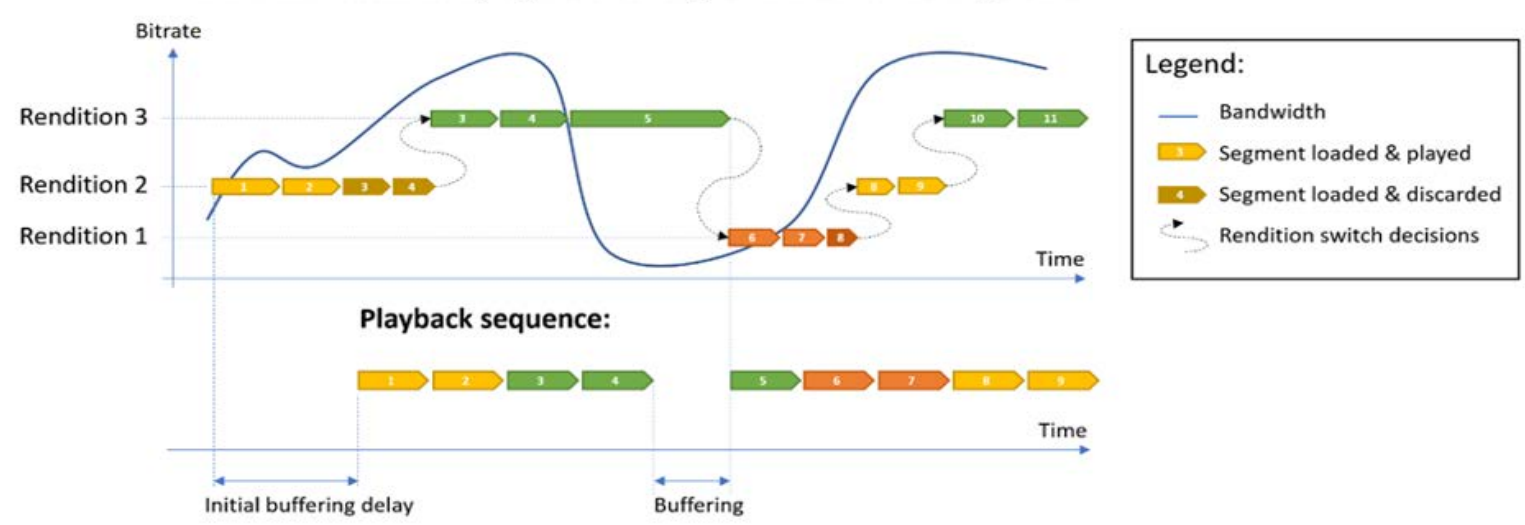

Figure 2: Illustration of segment loading, stream switching, playback and buffering events during streaming session.

streams selection logic, resulting in frequent buffering events, frequent switches, or delivering lower quality streams than possible for a given network. But all such phenomena can be discovered and understood only if there are datasets exposing them.

Therefore, to enable the research community to progress its work and have references to statistics of networks and client behaviors that are typical in today's practice, we have made several such data sets available in [8]. This paper describes characteristics of these data sets and their possible uses.

\subsection{Related work}

Most early studies on adaptive streaming systems have focused on network-related issues: congestions, packet losses, CND cache misses, etc. [1-3,9]. Rate adaptation algorithms have also been extensively studied [10-12,22-23]. The fact that content can be different has also been exploited, producing so-called "pertitle" [13], "content-aware" [14], and "context-aware" techniques [15-18]. Examples of uses of probabilistic techniques and simple client models can be found in [15,16,18]. A more complete client behavior model, capturing adaptation to both bandwidth and video player resolution has been proposed in [19].
References [20,21] discuss the analysis of the average-case behavior of streaming systems and some related optimization problems. The development of QoE metrics for streaming has also been an area of active research [25-32]. A recently published ITU$\mathrm{T}$ Recommendation P.1203 [28] is a recent example of progress achieved in this direction.

Several data sets related to various aspects of the operation of streaming systems have also been proposed. Reference [24] offers a data set of encoded content that can be used as inputs for streaming. References [26,27] describe a data set of encoded streams and associated MOS score values. This data set is used to train the QoE quality metric (VMAF). References [26-29] describe datasets that have been used in the course of the development ITUT P.1203 standard. These datasets include playback session characteristics as well as MOS scores measured by panels of viewers. These are again used to derive the QoE metric. 
Table 2: Illustration of segment loading, stream switching, playback and buffering events during streaming session.

\begin{tabular}{|c|c|c|}
\hline Category & Parameter & Description \\
\hline \multirow[t]{2}{*}{ session } & session & Randomly generated number associated with each player session. \\
\hline & seq & Sequential number of an event within a session \\
\hline \multirow[t]{6}{*}{ client } & device_type & Device type [desktop, mobile, tablet, tv, other] \\
\hline & device_os & OS type [windows, osx, linux, android, ios, webos, other] \\
\hline & browser & Browser type [chrome, firefox, safari, edge, ie, opera, other] \\
\hline & player & Player type [app - dedicated application, web - JS / browser-based player] \\
\hline & player_width & Player window width [pixels] \\
\hline & player_height & Player window height [pixels] \\
\hline \multirow[t]{8}{*}{ rendition } & rendition_indicated_bps & Rendition bitrate [bps]. Sum of audio and video bitrates. \\
\hline & rendition_width & Video width as encoded [pixels] \\
\hline & rendition_height & Video height as encoded [pixels] \\
\hline & rendition_framerate & Video framerate $[\mathrm{fps}]$ \\
\hline & video_codec & Video codec type [h264, hevc, av1] \\
\hline & video_codec_profile & Video codec profile [baseline, main, high] \\
\hline & format & Streaming format $\left[h l s \_v 3, h l s \_v 7\right.$, dash] \\
\hline & segment_duration & Segment duration [seconds] \\
\hline \multirow[t]{5}{*}{ playback } & video_seconds_viewed & Seconds of media content played in the period between the last two player events \\
\hline & forward_buffer_seconds & Number of seconds of media content buffered but not yet played \\
\hline & rebuffering_seconds & Total number of seconds the player was "buffering" in the period between the last two player events \\
\hline & rebuffering_count & Number of times the player was "buffering" in the period between the last two player events \\
\hline & media_bytes_transferred & Total number of bytes transferred since the start of the session \\
\hline network & measured_bps & Network bandwidth [bps] estimated based on size and delivery time of the last segment downloaded \\
\hline
\end{tabular}

An excellent review of various available data sets for streaming QoE measurements can also be found in [32]. However, all these datasets are focusing on measuring and assessing the final effect QoE of streaming, and not its connection to limiting factors - such as network statistics, player sizes, device characteristics, and other factors. In other words, such datasets are adequate for studies of QoE, but not the efficiency of the streaming system.

In this context, the data sets that we are proposing are more complete and enable the study of the problem more holistically. Our datasets include both network bandwidth measurements as available to players, as well as full traces of their decisions, as well as all media-coding-related parameters needed to assess QoE. These datasets have been collected based on a set of real-world large-scale streaming events, providing a large number of data points, enabling accurate statistical analysis.

\subsection{Outline}

This paper is organized as follows. In Section 2, we will describe the data sets that we made available. In Section 3. we offer sketches of possible applications of uses of these data sets. In Section 4, we will draw conclusions.

\section{STREAMING STATISTICS DATASETS}

Repository [8] includes data sets that we collected for 4 different streaming events. The overall characteristics of these events/datasets are summarized in Table 1.

The playback was done by a population of different devices; PCs, TVs, mobiles, tablets, etc. In most cases, the native (built-in) players have been utilized: AVplayer on Apple's iOS and OSXbased platforms, ExoPlayer-based players on Android devices, and respective native players on TV OSes. On PCs, streaming was done by open source video.js players [7].
Both DASH and HLS [4,5] standards were used for streaming. The number of encoded streams in each event varied from 4 to 5 , and so were the ranges of bitrates and resolutions.

The playback statistics were collected by Brightcove Analytics system [6]. The descriptions of all metrics present in the data sets are given in Table 2. Such metrics include information about streaming sessions, clients, renditions, playback statistics, and network behavior. Effectively for each session, we provide a log of all associated player events with such metrics reported. Player events were sent at periodicity of at most $10 \mathrm{sec}$. Separate events were also sent when player was switching between renditions. This way the values of video_seconds_viewed or media_bytes_transferred parameters can be associated with specific renditions that have been used.

Table 1: Summary statistic for events included in the dataset.

\begin{tabular}{lllll}
\hline Characteristic & Event I & Event II & Event III & Event IV \\
\hline Player events & $30,706,904$ & $10,006,501$ & 687,239 & $3,675,616$ \\
\hline Min. video bitrate [kbps] & 300 & 900 & 141 & 450 \\
\hline Max. video bitrate [kbps] & 1,000 & 4,040 & 1,750 & 2,100 \\
\hline Min. resolution [pixels] & $426 \times 240$ & $640 \times 360$ & $360 \times 202$ & $480 \times 270$ \\
\hline Max. resolution [pixels] & $960 \times 540$ & $1280 \times 720$ & $1280 \times 720$ & $1280 \times 720$ \\
\hline The number of renditions & 4 & 4 & 4 & 5 \\
\hline Streaming protocol & HLS, DASH & HLS, DASH & HLS & HLS \\
\hline Segment length [sec] & 4 & 4 & 6 & 10 \\
\hline Receiving devices & tv, & tv, & tv, desktop, & desktop \\
& desktop, & desktop, & mobile, & \\
& mobile, & mobile, & tablet, & \\
& tablet, & tablet, & other & \\
\hline other & other & & 22,341 \\
\hline Avg. bandwidth [kbps] & 27,969 & 56,053 & 30,400 & \\
\hline
\end{tabular}




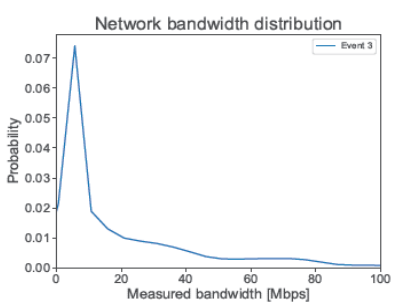

(a) PDF of network bandwidth

Percentage of device type

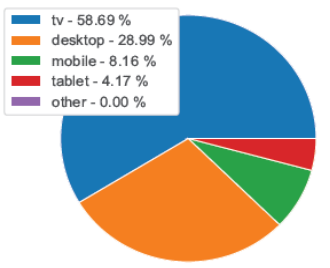

(c) Distribution of device type

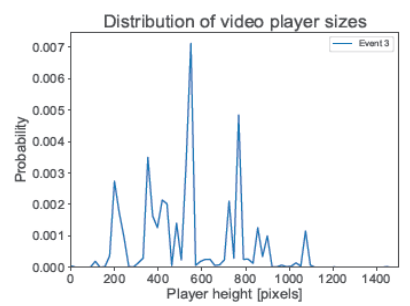

(b) PDF of rendered player width

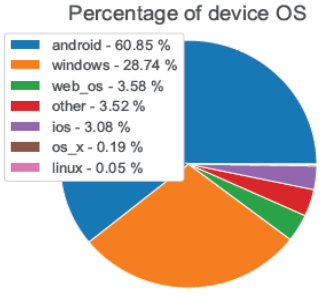

(d) Distribution of device OS

Figure 3: Network and usage statistics for the Event I.

Table 3: Encoding profile used for the Event I.

\begin{tabular}{cccccc}
\hline rendition & codec & profile & resolution & framerate & bitrate \\
\hline 0 & h.264 & baseline & $426 \times 240$ & 29.97 & 300 \\
1 & h.264 & baseline & $640 \times 360$ & 29.97 & 500 \\
2 & h.264 & baseline & $852 \times 480$ & 29.97 & 750 \\
3 & h. 264 & baseline & $960 \times 540$ & 29.97 & 1000 \\
\hline
\end{tabular}

To simplify parsing of the player event logs, we include two session-related parameters: unique session number, and the even sequence number in the session. Thus, by selecting all events for a particular session, and ordering them according to the sequence number it should be possible to restore order of events in player and then understand all decisions that it made during playback, and also see what could have possibly influenced it. The observed network bandwidth values are reported as measured_bps, and the effective sizes of video player window are reported as player_width and player_height respectively. The state of player's forward buffer at each point in time is reported by the forward_buffer_seconds parameter.

\subsection{Event I}

Table 3 presents the encoding profile used for Event I, comprising of four different renditions using H.264 baseline profile, maximizing device compatibility. The bitrate selection for rendition 0 of $300 \mathrm{Kbps}$ with a resolution of $426 \times 240$ pixels (width $\mathrm{x}$ height) provides an option for continuous playback in slower networks, while the smaller screen size adds support for lower-end handhelds. On the other end, rendition 3 targets faster networks with $1000 \mathrm{kbps}$ of available bandwidth with higher video quality.

As can be seen in Figure 3 (a), the most common measured bandwidth is under $15 \mathrm{Mbps}$ with player sizes being under 600 pixels cf. Figure 3 (b), which translates to profiles 0 and 1 . Due to internal $\mathrm{ABR}$ algorithm decisions, the selected profile in certain scenario might vary, i.e., even if the rendered player size is slightly

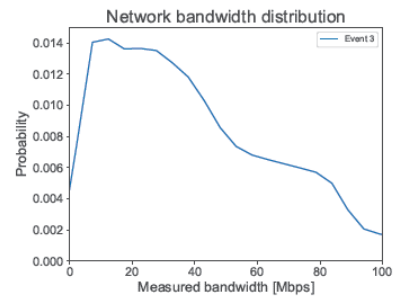

(a) PDF of network bandwidth Percentage of device type

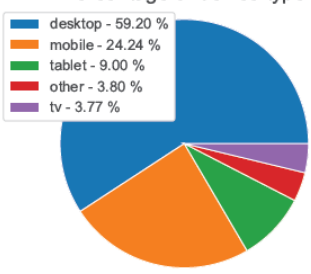

(c) Distribution of device type

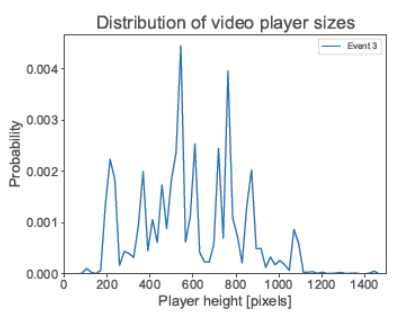

(b) PDF of rendered player width

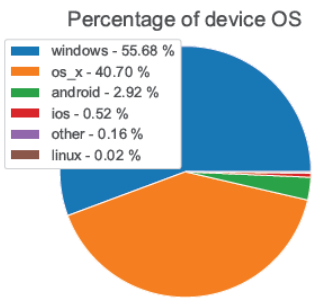

(d) Distribution of device OS
Figure 4: Network and usage statistics for the Event II.

Table 4: Encoding profile used for the Event II.

\begin{tabular}{cccccc}
\hline rendition & codec & profile & resolution & framerate & bitrate \\
\hline 0 & h. 264 & main & $640 \times 360$ & 30 & 900 \\
1 & h. 264 & main & $960 \times 540$ & 30 & 1700 \\
2 & h. 264 & high & $1280 \times 720$ & 30 & 2400 \\
3 & h. 264 & high & $1280 \times 720$ & 30 & 4040 \\
\hline
\end{tabular}

smaller than the rendition size, the player might choose to request a bigger rendition without compromising playback. Figures 3 (c) and (d) provide the breakdown of device type and device OS, respectively. With TV being the most popular device in this dataset, is not surprising that Android OS is also the most popular operating system, as it is used by most TV vendors. Additionally, $71.93 \%$ of the mobile market use Android OS. Windows desktops devices are the second most common entries.

\subsection{Event II}

Table 4 shows the encoding profiles for Event II. Four different renditions were generated using H.264 codec with main and high profiles, with bitrates varying from 900 to $4040 \mathrm{kbps}$. H.264 main profile is intended for newer devices with more computing power to decode the video (Android 6.0+ for instance2) while the high profile is intended for high-definition streaming. In this case, there are two renditions with the same size $(1280 \times 720)$; however, different bitrates were used to accommodate multiple network characteristics.

Figure 4 (a) depicts the measured network bandwidth for the event, while Figure 4 (b) shows the player size rendered on the screen. It is worth noting the wider distribution of both bandwidths, with a long tail that extends to $1000 \mathrm{Mbps}$ (bandwidth greater than 


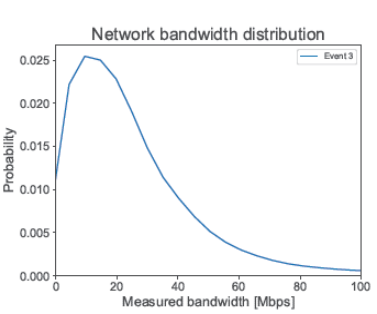

(a) PDF of network bandwidth Percentage of device type

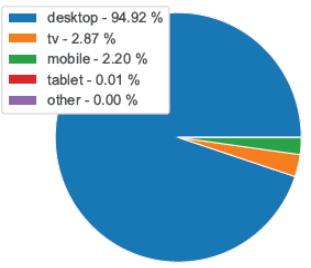

(c) Distribution of device type

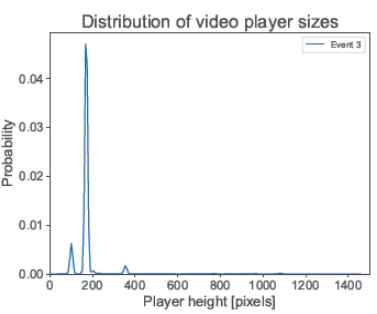

(b) PDF of rendered player width Percentage of device OS

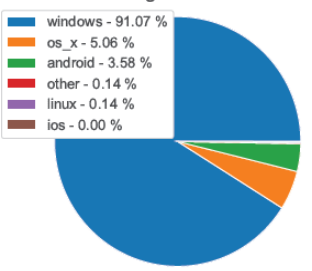

(d) Distribution of device OS

Figure 5: Network and usage statistics for the Event III.

Table 5: Encoding profile used for the Event III.

\begin{tabular}{cccccc}
\hline rendition & codec & profile & resolution & framerate & bitrate \\
\hline 0 & h.264 & baseline & $360 \times 202$ & 29.97 & 141 \\
1 & h. 264 & baseline & $480 \times 270$ & 29.97 & 300 \\
2 & h. 264 & main & $640 \times 360$ & 29.97 & 770 \\
3 & h. 264 & main & $1280 \times 720$ & 29.97 & 1750 \\
\hline
\end{tabular}

$100 \mathrm{Mbps}$ are not shown to facilitate visibility across events, but can be easily plotted), and player sizes compared to Event I, which indicates that viewers have better access to up-to-date devices and faster networks. Figures 4 (c) and 4 (d) show that the majority of users watched the stream on Windows or Mac OS (more than $95 \%$ combined) either on mobile devices or desktops.

\subsection{Event III}

The encoding information for Event III is provided by Table 5, comprising of H.264 baseline and main profiles. The four profiles offer a wide range of bitrates, with $141 \mathrm{kbps}$ for rendition 0 to 1750 kbps for rendition 3, adding support for multiple devices and networks. As shown in Figure 5 (b), the most common player size is about 200 pixels in height, with the majority of viewers watching on Windows desktops (Figures 5 (c) and (d)). This shows that the player was embedded in a web page, and not expanded to full screen often. As can be seen in Figure 5 (a), there is enough available bandwidth to retrieve high-bitrate renditions (rendition 2 and 3, for instance); however, due to the player being rendered with a smaller size, the ABR algorithm choose not to request these renditions.

\subsection{Event IV}

Table 6 summarizes the encoding profiles for Event IV, which provides five different renditions, covering the range of resolutions from $270 \mathrm{p}$ to $720 \mathrm{p}$, and range of bitrates from $450 \mathrm{Kbps}$ to $2100 \mathrm{Kbps}$.

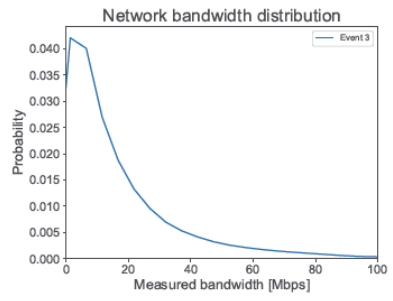

(a) PDF of network bandwidth Percentage of device type

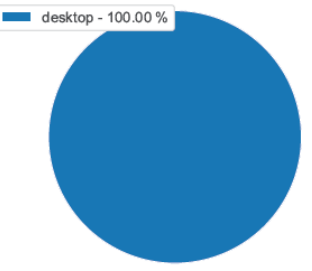

(c) Distribution of device type

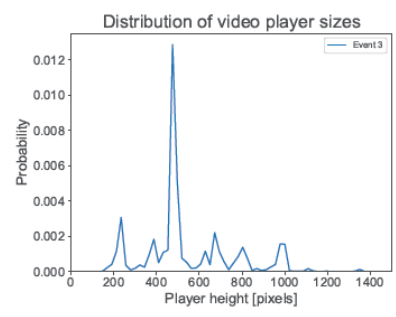

(b) PDF of rendered player width

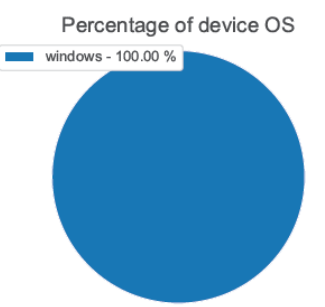

(d) Distribution of device OS
Figure 6: Network and usage statistics for the Event IV.

Table 6: Encoding profile used for the Event IV.

\begin{tabular}{cccccc}
\hline rendition & codec & profile & resolution & framerate & bitrate \\
\hline 0 & h. 264 & baseline & $480 \times 270$ & 23.976 & 450 \\
1 & h.264 & baseline & $640 \times 360$ & 23.976 & 800 \\
2 & h.264 & main & $768 \times 432$ & 23.976 & 1000 \\
3 & h.264 & main & $1024 \times 576$ & 23.976 & 1500 \\
4 & h.264 & main & $1280 \times 720$ & 23.976 & 2100 \\
\hline
\end{tabular}

Event IV data comprises of only Windows desktops, as can be seen in Figures 6 (c) and (d). The measured network bandwidth and player height, depicted in Figures 6 (a) and (b), respectively, show that the most common rendered player height is between 400 and 500 pixels high (extending past 1000 pixels high) with the most common bandwidth being under $20 \mathrm{Mbps}$. These characteristics are well suitable for players to request any of the renditions in the profile. It is worth noting that Event IV used a segment duration of 10 seconds, 2 which helps the player calculate more accurate network bandwidth.

\section{EXAMPLES USES OF THE DATASETS}

In this section we discuss several possible uses of the proposed datasets.

\subsection{Modeling the behavior of the clients}

First, by using these data sets it could be possible to arrive at models describing behavior of streaming clients.

For instance, in our recent paper [21], we proposed a simple model of streaming client deriving index of rendition being selected as function of 2 parameters:

$$
i\left(B, H_{p}\right)
$$

where $\mathrm{B}$ is a network bandwidth, and $H_{p}$ is the size of the video player window. This model has few tuning parameters, which can be established by fitting this model to the dataset. 


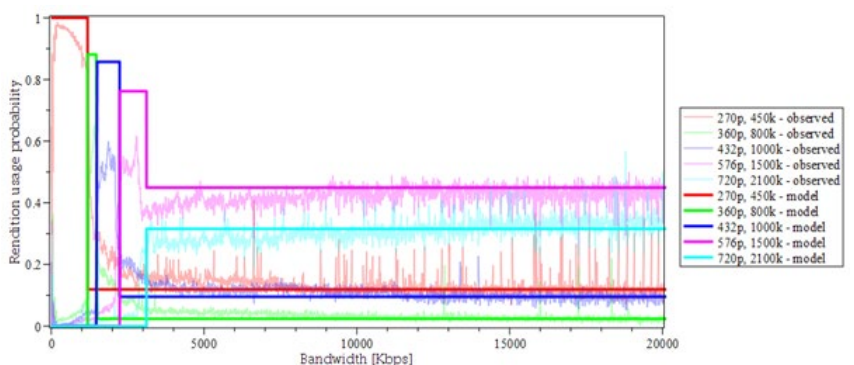

Figure 7: Observed vs actual client behavior in Event IV.

As an example, in Figure 7, we show a fit of the model [21] to experimentally obtained values of conditional probabilities of selection of each rendition based on our dataset for event IV. The depicted in Figure 7 conditional rendition selection probabilities are computed as follows:

$$
P(k \mid B)=\sum_{H_{p}: i\left(B, H_{p}\right)=k} q\left(H_{p}\right), \quad k=1, \ldots, n
$$

where $q\left(H_{p}\right)$ is the distribution of player sizes exhibited by the dataset. Based on Figure 7, we notice, that the proposed model fits experimentally observed data reasonably well.

\subsection{Modeling the performance of the system}

Once client model is established, it can be immediately used to derive the expressions for many average performance parameters of streaming systems.

For example, the average bitrate consumed by the streaming system can be expressed as follows:

$$
\bar{R}=\int_{0}^{\infty} p(B) \sum_{H_{p} \in \mathcal{H}_{p}} q\left(H_{p}\right) R_{i\left(B, H_{p}\right)} d B,
$$

where $p(B)$ is the distribution of the network bandwidth, $R_{i}$ denotes bitrate of rendition selected given each combination of bandwidth $B$ and player size $H_{p}$ and where integration and summation are done for both.

Similarly, we can produce expression for the average quality achieved in the system:

$$
\bar{Q}=\int_{0}^{\infty} p(B) \sum_{H_{p} \in \mathcal{H}_{p}} q\left(H_{p}\right) Q\left(H_{i\left(B, H_{p}\right)}, H_{p}, R_{i\left(B, H_{p}\right)}\right) d B,
$$

where $Q\left(H_{i}, H_{p}, R_{i}\right)$ is a model of quality for encoded and delivered stream, given its encoded resolution $H_{i}$, bitrate $R_{i}$, and player size $H_{p}$. Additional details about suitable for this purpose quality metric and performance analysis of streaming systems can be found in [21].

As evident, such formulae require known distributions for network bandwidth $p(B)$, player sizes $q\left(H_{p}\right)$, as well as client model $i\left(B, H_{p}\right)$, which all can be derived based on statistics that we provide in our datasets.

\section{CONCLUSIONS}

In this paper we have proposed dataset capturing statistics of several large-scale real-world streaming events, with diverse set of characteristics in terms of networks and types of receiving devices/players. Possible uses of these data sets have been discussed. The data sets are currently available in [8]. We hope they will be useful to the research community.

\section{REFERENCES}

[1] D. Wu, Y.T. Hou, W. Zhu, Y-Q. Zhang, and J.M. Peha, "Streaming video over the internet: approaches and directions," IEEE Trans. CSVT, vol. 11, no. 3, pp. 282-300, 2001.

[2] B. Girod, M. Kalman, Y.J. Liang, and R. Zhang, "Advances in channel-adaptive video streaming," Wireless Comm. and Mobile Comp., vol. 2, no. 6, pp. 573-584, 2002.

[3] G. J. Conklin, G. S. Greenbaum, K. O. Lillevold, A. F. Lippman, and Y. A. Reznik, "Video coding for streaming media delivery on the internet," IEEE Trans. CSVT, vol. 11, no. 3, pp. 269-281, 2001.

[4] R. Pantos, and W. May, "HTTP live streaming, RFC 8216," https://tools.ietf.org/html/rfc8216, 2017.

[5] ISO/IEC 23009-1:2012, "Information technology - Dynamic adaptive streaming over HTTP (DASH) - Part 1: Media presentation description and segment formats," February 2012.

[6] Brightcove VideoCloud platform https://www.brightcove.com/en/online-videoplatform

[7] Video.js open-source project, https://github.com/videojs/video.js

[8] Brightcove data set. https://github.com/brightcove/streaming-dataset

[9] D. Lee, C. Dovrolis, A. Begen, "Caching in HTTP Adaptive Streaming: Friend or Foe?," in Proc. ACM Network and Operating System Support on Digital Audio and Video Workshop, 2014, pp. 31-36.

[10] S. Hesse, "Design of scheduling and rate-adaptation algorithms for adaptive HTTP streaming," in Proc. SPIE 8856, Applications of Digital Image Processing XXXVI, 88560M, 2013

[11] C. Zhou, X. Zhang, L. Huo, and Z. Guo, "A control-theoretic approach to rate adaptation for dynamic HTTP streaming," in Proc. Visual Comm. Image Processing, San Diego, CA, 2012, pp. 1-6.

[12] K. Spiteri, R. Urgaonkar, R. K. Sitaraman, BOLA: Near-Optimal Bitrate Adaptation for Online Videos. IEEE/ACM Trans. Netw. 28(4): 1698-1711 (2020)

[13] A. Aaron, et al, "Per-title encode optimization," https://medium.com/netflixtechblog/per-title-encode-optimization-7e99442b62a2, Dec. 152015.

[14] UltraHD Forum, "UltraHD Forum phase B guidelines," https://ultrahdforum.org/wp-content/uploads/ Ultra-HD-Forum-Phase-BGuidelines-v1.0.pdf, April 2018.

[15] Y. Reznik, K. O. Lillevold, A. Jagannath, J. Greer, and J. Corley, "Optimal design of encoding profiles for ABR streaming," in Proc. Packet Video Workshop, Amsterdam, NL, June 12, 2018, pp. 43-47.

[16] Y. Reznik, X. Li, K. O. Lillevold, A. Jagannath, and J. Greer, "Optimal MultiCodec Adaptive Bitrate Streaming," in Proc. IEEE Int. Conf. Multimedia \& Expo, Shanghai, China, 2019, pp. 348-353.

[17] Y. Reznik, X. Li, K.O. Lillevold, R. Peck, T. Shutt, and P. Howard, "Optimizing Mass-Scale Multi-Screen Video Delivery," SMPTE Motion Imaging Journal, vol. 129, no. 3, pp. 26-38, April 2020.

[18] C. Chen, Y. Lin, S. Benting, and A. Kokaram, "Optimized transcoding for large scale adaptive streaming using playback statistics," in Proc. IEEE Int. Conf. Image Proc., Oct 2018, pp. 3269-3273.

[19] Y. Reznik, K. Lillevold, A. Jagannath, and X. Li, "Towards Understanding of the Behavior of Web Streaming," Proc. The Picture Coding Symposium (PCS'21) Bristol, UK, June 29 - July 2, 2021.

[20] Y. Reznik, K. Lillevold, R. Vanam, "Perceptually optimized ABR ladder generation for Web streaming," Proc. IS\&T Electronic Imaging, San Francisco, CA, January 18-21, 2021.

[21] Y. Reznik, "Average Performance of Adaptive Streaming," Proc. Data Compression Conference (DCC'21), Snowbird, UT, 23-26 March 2021.

[22] T-Y. Huang, N. Handigol, B. Heller, N. McKeown, and R. Johari, "Confused, Timid, and Unstable: Picking a Video Streaming Rate is Hard," in Proc. Internet Measurement Conference (IMC'12), Boston, Massachusetts, USA, 2012, pp. 225-238.

[23] S.S. Krishnan, and R. K Sitaraman, "Video stream quality impacts viewer behavior: inferring causality using quasi-experimental designs," IEEE/ACM Transactions on Networking, vol. 21, no. 6, 2013, pp. 2001-2014. 
[24] S. Lederer, C. Müller, and C. Timmerer, "Dynamic Adaptive Streaming over HTTP Dataset," in Proc. ACM Multimedia Systems Conference (MMSys '12), Chapel Hill, North Carolina, 2012, pp.89-94.

[25] B. Belmudez and S. Moller, "An approach for modeling the effects of video resolution and size on the perceived visual quality", in Proc IEEE Int. Symp. Multimedia, Dana Point CA, 2011, pp. 464-469.

[26] Z. Li, A. Aaron, et al., Toward A Practical Perceptual Video Quality Metric, June 2016, URL: https://netflixtechblog.com/toward-a-practical-perceptual-videoquality-metric-653f208b9652

[27] Netflix dataset:

https://drive.google.com/drive/folders/0B3YWNICYMBIweGdJbERIUG9zc0k

[28] ITU-T Recommendation P.1203: Parametric bitstream-based quality assessment of progressive download and adaptive audiovisual streaming services over reliable transport", October, 2017.

[29] W. Robitza, M.-N. Garcia, and A. Raake, "A modular HTTP adaptive streaming QoE model - Candidate for ITU-T P.1203 (P.NATS)," in Proc. 9th Int. Conf Qual. Multimedia Exper. (QoMEX), Erfurt, Germany, May/Jun. 2017, pp. 1-6.

[30] S. Satti, C. Schmidmer, M. Obermann, R. Bitto, L. Agarwal, and M. Keyhl, "P.1203 evaluation of real OTT video services," in Proc 9th Int. Conf. Qual. Multimedia Exper. (QoMEX), Erfurt, Germany, May/Jun. 2017, pp. 1-3.

[31] W. Robitza et al., "HTTP adaptive streaming QoE estimation with ITU-T Rec. P. 1203: Open databases and software," in Proc. 9th ACM Multimedia Syst. Conf., Amsterdam, The Netherlands, 2018, pp. 466-471.

[32] N. Barman and M. G. Martini, "QoE Modeling for HTTP Adaptive Video Streaming-A Survey and Open Challenges," in IEEE Access, vol. 7, 2019, pp. 30831-30859. 